\title{
Clinical Trial to Analyze the Effect of Oral Intake of Phellinus Linteus(Sanghuang) Extract on Immune Function: A Study Protocol for a Randomized, Double-blind Controlled Trial
}

\section{Yong Ho Ku}

Daejeon university https://orcid.org/0000-0001-7553-4144

Hyun Lee

Daejeon University College of Oriental Medicine: Daejeon University College of Korean Medicine Hwa Yeon Ryu

Daejeon University College of Oriental Medicine: Daejeon University College of Korean Medicine Jae Hui Kang ( $\nabla$ rkd12@hanmail.net)

Department of Acupuncture \& Moxibustion Medicine, College of Korean Medicine, Daejeon University https://orcid.org/0000-0002-2988-7624

\section{Study protocol}

Keywords: immune function, Phellinus linteus, randomized controlled trial

Posted Date: June 30th, 2021

DOI: https://doi.org/10.21203/rs.3.rs-654244/v1

License: (9) This work is licensed under a Creative Commons Attribution 4.0 International License. Read Full License

Version of Record: A version of this preprint was published at Trials on November 27th, 2021. See the published version at https://doi.org/10.1186/s13063-021-05740-5. 


\section{Abstract}

Background: As Korea becomes an aging society, interest in health care has increased. In particular, there is an increasing demand for immune function improvement to prevent infectious diseases. Phellinus linteus (PL) has previously been shown to have immune-enhancing and anticancer effects. We aim to evaluate whether PL mycelium extract, cultured using the PL KCTC0399BP strain, has an increase in immune function using blood test indicators. This clinical trial is designed based on the results of a pilot study as the main trial.

Methods: This clinical trial will be a randomized, double-blinded, placebo-controlled trial. Ninety-eight participants will be enrolled and randomly divided into two groups: experimental group (PL $1000 \mathrm{mg}$ ) and control group (placebo). Participants will be administered experimental food or placebo for 8 weeks. Blood tests will be performed before food intake and at 8 weeks after the start of the experiment. Laboratory evaluation items are as follows: natural killer cell activity, tumor necrosis factor-a, interferon- $\gamma$, interleukin (IL)-1 $\beta$, IL-2, IL-6, IL-12, immunoglobulin (Ig)G1, IgG2, and IgM. We will mainly use the full analysis set to statistically analyze the effectiveness of treatment.

Discussion: This study includes inclusion and exclusion criteria and a well-controlled intervention. This study evaluates the effect of PL extract on immune function and will contribute to knowledge on the value of $\mathrm{PL}$ as an immune function health functional food.

Clinical research registration: This study has been registered at the Clinical Research Information Service (CRIS) of Korea: CRIS-KCT0005460. Registered , 12 October 2020, https://cris.nih.go.kr/cris/search/search_result_st01_en.jsp?seq=17761\&ltype=\&rtype=

Trial status: This clinical trial is in the recruitment stage. Recruitment began in October 21, 2020 and will be completed in March 2021. This trial was registered at the Clinical Research Information Service of South Korea on October 12, 2020. (CRIS-KCT0005460)

\section{Introduction}

There is a growing interest in health globally, and the demand for medicines and foods related to immune function increases with aging and the spread of infectious diseases. Immunity is a means of defense that protects our bodies from external influences. From mild diseases such as colds to infectious diseases and cancer, immunity is a key factor in various diseases.

The immune-improving function of Phellinus linteus (PL) is known, and it has been reported to increase the activity of interleukin (IL)-12, interferon (IFN)-y, and natural killer (NK) cells. [1,2] In a previous study, it was reported that PL extract increases immunity mediated by cells such as T-lymphocytes, NK cells, macrophages, and B-lymphocytes. [3] In a recent study, the antitumor and antioxidant activities of PL were reported. [4] 
In a patent using the existing PL KCCM KSSW01 strain, the immunity enhancing effect of glucomannan contained in PL extract was proven. [5] Glucomannan consists of $53.7 \%$ glucose, $23.5 \%$ mannose, $7.7 \%$ galactose, $7.1 \%$ xylose, $5.8 \%$ fructose, and 2.2\%, 3-0-methylglucose. The PL extract used in this study contains galactomannoglucan using the PL KCTC0399BP strain. Galactomannoglucan comprises $78.6 \%$ glucose, $18.0 \%$ mannose, and $3.4 \%$ galactose, which is different from the existing glucomannan. [6]

This clinical trial aims to analyze and evaluate whether PL extract is effective in improving immune function as an ingredient of health functional food. This clinical trial is the main trial and is designed to confirm efficacy and stability based on the results of a pilot study.

\section{Methods}

\subsection{Study design}

This is a prospective, double-blind, single-center, randomized clinical trial designed to demonstrate the efficacy of PL extract on immune function. A total of 98 subjects satisfying the inclusion and exclusion criteria are enrolled at the Daejeon University Cheonan Korean Medicine Hospital. Recruitment started in October 2020, and the clinical trial is expected to end in March 2021. Subjects will be recruited through placards on outdoor billboards and banners in hospitals. All subjects received written explanations and informed consent forms from the Korean medicine doctor (KMD) for the clinical trial protocol.

The 98 subjects who participated in this clinical trial have been randomly assigned 1:1 to either the experimental group (PL $1000 \mathrm{mg}$ ) or the control group (placebo). Subjects will take 1 capsule of $500 \mathrm{mg}$ twice daily for 8 weeks, and depending on the group assigned ingest either PL 1000 mg or dextrin 1000 mg daily.

The evaluation variables are NK cell activity, tumor necrosis factor (TNF)-a, IFN- - , IL-1 $1 \beta$, IL-2, IL-6, IL-12, immunoglobin $(\mathrm{lg}) \mathrm{G} 1, \mathrm{lg} \mathrm{g} 2$, and $\mathrm{IgM}$. A laboratory test will be conducted on the 0 th day before taking the test food, and after 8 weeks at the end of the test. The subjects will visit thrice, and the clinical trial spans a total of 8 weeks. (Table 1, Figure 1)

\subsection{Inclusion and exclusion criteria}

The inclusion criteria are as follows: men and women aged 20-65 years, those with peripheral white blood cells between $3 \times 10^{3} / \mu \mathrm{L}$ and $10 \times 10^{3} / \mu \mathrm{L}$, having upper respiratory infections or history of twice or more upper respiratory infections within 1 year before the start of the test, those who have had symptoms of a cold, and those who have agreed to participate in this test and have signed (the subject or legal representative) a written consent form voluntarily.

The exclusion criteria are as follows: clinically significant acute or chronic cardio-cerebrovascular system, immune system, respiratory system, hepatobiliary system, kidney and urinary system, and nervous system; currently being treated for musculoskeletal disease and psychotic, infectious, and 
hematologic/neoplastic diseases; persons (however, considering the condition of the subject, they can participate in the study at the discretion of the research director) with uncontrolled hypertension (160/100 $\mathrm{mmHg}$ or more, measured after $10 \mathrm{~min}$ of rest for the subject), uncontrolled diabetes (fasting blood glucose $126 \mathrm{mg} / \mathrm{dL}$ or higher, or if a new drug is started owing to diabetes within 3 months), AST (GOT) or ALT (GPT) blood level is 3-fold or more than the normal upper limit of the relevant institution, and creatinine blood level is above $2.4 \mathrm{mg} / \mathrm{dL}$ in men and exceeding $1.8 \mathrm{mg} / \mathrm{dL}$ in females; those who ingested or are currently ingesting functional foods for health that may affect immunity within 2 weeks before the screening; those who complain of severe gastrointestinal symptoms such as heartburn and indigestion; pregnant, lactating, or plan to become pregnant during the study period; those who are sensitive or allergic to test food-related foods; those who plan to participate in other studies during this study and those who have received other study drugs within 4 weeks of starting the study; and those who the researcher deemed unsuitable for this study

\subsection{Dosage calculation}

A dose of $200 \mathrm{mg} / \mathrm{kg}$ administered to mice for 1 week did not show any specific toxicity. In the animal test, the effective dose of $200 \mathrm{mg} / \mathrm{kg}$ was calculated based on an adult body weight of $60 \mathrm{~kg}$ using a conversion factor of 0.08 , compared to body surface area, and the human intake dose was set to 960 $\mathrm{mg} /(\mathrm{kg}$ day). In a preliminary clinical study, when PL extract was ingested at 1000 and $2000 \mathrm{mg} /$ day, no adverse reactions related to the test food were observed, and it was confirmed that the immune function was improved at $1000 \mathrm{mg} /$ day. Therefore, the PL extract intake was set to $1000 \mathrm{mg} / \mathrm{day}$ based on the results of preliminary clinical studies and animal experiments.

\subsection{Sample size calculation}

The sample size was calculated using the results of NK cell activity, an efficacy evaluation variable in the exploratory clinical trial. Calculations were performed using G*power 3.1.9.4, and the minimum number of subjects to confirm statistical significance was determined as 41 individuals per group. Considering a dropout rate of $15 \%$, we aimed to recruit a total of 98 people, 49 for each group.

\subsection{Randomization and blinding procedures}

The final registered subjects have been assigned 1:1 to the experimental group and control group using block randomization. The total number of random assignments was generated on a scale of approximately $120 \%$ of the target recruitment number. A 3-digit randomized number was assigned according to randomization, and a test food or placebo was distributed to the subjects according to the number. If a randomized number is assigned, this randomized number cannot be reused even if the subject drops out. Researchers and subjects were blinded. Randomization details were generated by statistical experts using nQuery Advisor 7.0 (or SAS 9.0 or SPSS 21.0). Pharmaceutical companies put each subject's random assignment details in a non-permeable bag, sealed it, and supplied it to the supervisor for storage and management. 


\subsection{Intervention}

The clinical trial food was obtained from Hankookshinyak Pharmaceutical Co. (Nonsan, Republic of Korea). Test food and placebo had the same appearance and properties; therefore, no difference was observed with the naked eye, and there was no difference in weight. In addition, the same label was attached to keep the researchers and subjects blind.

Subjects will ingest 1 capsule twice a day 30 min after meals, for 8 weeks. Treatment received at the time of participation in the clinical trial, health functional foods to be taken, and additional treatments are considered as combination therapy. If co-administration is necessary, the administration details will be recorded in the case report form (CRF) and the treatment chart, and the possible combination therapy will not be changed. Concomitant drug administration should be minimized while participating in a clinical trial. Concomitant drugs will be administered according to the judgment of the person in charge of the clinical trial only when it is judged that it does not affect the clinical trial. If a drug that directly affects immune function is taken, the subject should stop the clinical trial.

\subsection{Outcome measures}

The primary outcome is the amount of change in NK cell activity. NK cells play a key role in immune function against viruses and have recently been shown to play an important role in anticancer activity. [7] NK cell activity is measured to evaluate whether immune function has increased. The secondary outcome is the amount of change in white blood cells in peripheral blood, and in the levels of TNF-a, IFN- $\gamma$, IL-1 $\beta$, IL2, IL-6, IL-12, IgG1, IgG2, and IgM. Proinflammatory cytokines such as TNF- $a$, IFN- $\gamma$, IL-1 $\beta$, IL-2, IL-6, and IL12 are factors that regulate the inflammatory response of tissues. [8] Immunoglobulins such as IgG1, $\lg \mathrm{G} 2$, and $\lg \mathrm{M}$ are involved in virus neutralization and aggregation reactions. They also play a major role in immunity against influenza. [9] Immune function can be confirmed through changes in cytokines and immunoglobulins.

\subsection{Data collection and monitoring}

Outcome measures will be measured before administration of the test food (visit 1) and after 8 weeks at the end of administration (visit 3 ) through a lab test. Personal information is examined during screening. Monitoring is to be performed by the LAB to MEDI CRO Co., Ltd. (Seoul, South Korea). During monitoring, research plans, case records, and annexed documents will be reviewed to check whether they are scientifically performed according to the plans approved by the IRB, and to ensure that the safety and rights of the research subjects are not infringed.

\subsection{Statistical analysis}

Demographic data measured by screening will be tested using the $t$-test (or Wilcoxon rank-sum test) for continuous data. For categorical data, a chi-square test (or Fisher's exact test) will be performed. Effectiveness evaluation is based on the results of the full analysis set analysis. The per protocol (PP) group will also be analyzed for reference. Statistical significance is determined at a significance level of 
$5 \%$. If the difference between the two groups shows a normal distribution, an independent $t$-test will be performed. Otherwise, the Mann-Whitney's $U$ test will be performed. For the test of difference before and after the dose within a group, a paired $t$-test will be performed when the data are normally distributed, else, a Wilcoxon's signed-rank test will be performed. The Shapiro-Wilk's test will be performed to test the normality of continuous variables. When a difference between groups is observed, the drinking history variable will be considered as a control variable, and covariate analysis will be performed additionally. In the full analysis set analysis, the treatment of missing values is to be replaced by the last observation carried forward. In safety evaluation, the $95 \%$ confidence interval within the group is to be determined by calculating the rate of occurrence of adverse reactions, and comparisons will be made between groups. From the results of each lab test, continuous data such as vital signs will be compared with the baseline and analyzed using either a paired $t$-test or $t$-test. If normality is not satisfied, the Wilcoxon signed-rank test or Wilcoxon rank-sum test will be performed. Categorical data will be analyzed using the chi-square test and Fisher's exact test to determine whether there is a difference between groups by presenting the frequency and ratio of each category.

\subsection{Withdrawal and dropout}

Subjects who have been fed food from the clinical trial for 8 weeks will be considered to have completed this clinical trial. Subjects may be dropped in the following cases: violation of inclusion/exclusion criteria (limited to screening), request to stop the test by the study subject, if a serious adverse reaction occurs in the subject of the human application test or if it is difficult to continue the human clinical trial owing to the adverse reaction, a major violation of the human study protocol when the use of prohibited drugs can affect the value of the efficacy evaluation variable, the subject's food consumption is either noncompliant (less than $80 \%$ compliance) or unobservable, those who have taken or must take medicines or health functional foods that may affect this human clinical trial, and if the subject is pregnant during the human clinical trial period, based on the researchers' judgment.

All causes of dropout should be documented in detail in the CRF. A laboratory test will also be performed to evaluate the possible safety of the subjects who dropped out. If an adverse event (AE) occurs, followup observation will be conducted and reported until the cause is identified. In case of dropout owing to a serious adverse reaction, the IRB shall be notified immediately.

\subsection{Safety}

Side effects such as AEs, adverse drug reactions, and serious adverse events (SAEs) will be checked at the time points of Visit 2 and 3. When an AE occurs, it will be classified and evaluated as mild, moderate, or severe. If an SAE occurs, it will be reported to the IRB within $24 \mathrm{~h}$ of recognition. The IRB shall review the report and develop appropriate follow-up measures. The clinical trial implementation organization will make every effort to protect the subject's safety. The clinical trial manager shall stop the clinical trial of the subject and take appropriate measures such as withdrawal if SAE occurs. If necessary, the blind can be removed. Safety evaluation shall be conducted using the rate of occurrence of side effects. The subject will be compensated for damages caused directly by the test food in accordance with the policy. 


\subsection{Ethics}

This clinical trial design is based on the Helsinki Declaration and the Korean Clinical Practice Guidelines. This clinical trial protocol has been approved by the institutional review boards of the Daejeon University Cheonan Korean Medicine hospital (DJUMC-2020-BM-07). If necessary, the protocol can be changed with permission from the IRB. This protocol has been registered with the Clinical Research Information Service of South Korea (CRIS-KCT0005460).

\section{Discussion}

The importance of the immune function is emphasized as the spread of infectious diseases increases worldwide in modern society. The immune system comprises various cytokines, immunoglobulins, and phagocytic cells to protect our body from external intruders. Infectious diseases related to the immune system are fatal in the elderly population with reduced immune function. Therefore, in modern society, where the elderly population increases, the demand for foods that can increase the immune function increases.

PL has an anticancer effect by improving immune function. [10] Other studies have reported the antiinflammatory effects of PL extract in rats, [11] anti-inflammatory mechanisms of PL in macrophages, [12] and antioxidant and antimicrobial activities of PL. [13] NK cells, responsible for innate immunity, play an important role in immune functions such as anticancer [14] and antiviral. [15] In the health functional food functional evaluation guide distributed by the Ministry of Food and Drug Safety in South Korea, NK cell activity should be evaluated using flow cytometry or the lactate dehydrogenase cytotoxicity assay kit by culturing effector and target cells together. The lactate dehydrogenase assay is a method of measuring cell death, and it evaluates the activity of NK cells by analyzing NK cell cytotoxicity. [16] In existing clinical trials, a diagnostic kit is used to measure NK cell activity. The kit artificially activates NK cells using engineered recombinant cytokines and then measures NK cell activity through IFN-y detection. The method using the kit has limitations in the analysis because it is not possible to know the NK cell activity using the effector:target concentration. In this clinical trial, NK cell activity will be confirmed using the lactate dehydrogenase assay at various concentrations of effector:target cells (e.g. 12.5:1, 25:1, and $50: 1)$. In this clinical trial, changes in NK cell activity, proinflammatory cytokines, and Igs will be checked to confirm the improvement in the immune function owing to PL extract. This clinical trial will verify the efficacy of PL extract as a health supplement by proving the effect of improved immune function.

\section{List Of Abbreviations}

PL, Phellinus linteus; NK, natural killer; TNF, tumor necrosis factor; IFN, interferon; Ig, immunoglobulin; IL, interleukin

\section{Declarations}


Ethical statement: This trial has been approved by the Institutional Review Board of the Daejeon University Cheonan Korean Medicine hospital. (DJUMC-2020-BM-07)

Consent for publication: Not applicable.

Availability of date and materials: The data of this study will be provided upon reasonable request. KMD participating in clinical trials will have access to the final trial dataset. There is no contractual agreements that limit such access for investigators.

Competing interests: The authors declare that they have no competing interests.

Funding: This study is supported by the Daejeon University Research Grants (2020). This study received funding from Daejeon University for publication as a Investigator initiated clinical trial (ITT).

Author's Contributions: The clinical trial protocol was designed, managed, supervised, and modified by JHK. YHK was in charge of developing the protocol and writing the manuscript. YHK, HYR examined the inclusion criteria in clinical practice. $\mathrm{HL}$, JHK critically revised the manuscript. JHK organized all procedure and revised the protocol. All authors have verified and approved the final manuscript.

Acknowledgements: This study is supported by the Daejeon University Research Grants (2020).

Author Contributions: The clinical trial protocol was designed, managed, supervised, and modified by JHK. YHK was in charge of developing the protocol and writing the manuscript. YHK, HYR examined the inclusion criteria in clinical practice. $\mathrm{HL}$, JHK critically revised the manuscript. JHK organized all procedure and revised the protocol. All authors have verified and approved the final manuscript.

Ethics approval and consent to participate: The clinical trial protocol has been reviewed and approved by the Institutional Review Board (IRB) of the Daejeon University Cheonan Korean Medicine hospital. (DJUMC-2020-BM-07) Protocol ver. 1.1, dated 14, September, 2020.

Funding: This study is supported by the Daejeon University Research Grants (2020). This study received funding from Daejeon University for publication as a Investigator initiated clinical trial (ITT).

The authors have no conflicts of interest to disclose.

\section{References}

[1] Kim GY, Oh WK, Shin BC, Shin YO, Park YC, Ahn SC, Lee JD, Bae YS, Kwak JY, Park YM. Proteoglycan isolated from Phellinus linteus inhibits tumor growth through mechanisms leading to an activation of CD11c+CD8+ DC and type I helper T cell-dominant immune state. FEBS Letters. 2004;76(3):391-400.

[2] Eom SY, Zhang YW, Kim NS, Kang JW, Hahn YS, Shin KS, Song HG, Park SY, Kim JS, Kim H, Kim YD. Effects of Keumsa Sangwhang (Phellinus linteus) Mushroom Extracts on the Natural Killer Cell Activity in Human. Korean Journal of Food Science and Technology. 2006;38(5):717-719. 
[3] Kim HM, Han SB, Oh GT, Kim YH, Hong DH, Hong ND, Yoo ID. Stimulation of humoral and cell mediated immunity by polysaccharide from mushroom Phellinus linteus. Int. J. Immunopharm. 1996;18:295-303.

[4] Lee JW, Back SJ, Bae WC, Park JM, Kim YS.Antitumor and Antioxidant Activities of the Extracts from Fruiting Body of Phellinus linteus. Mycobiology. 2006;34(4):230-235.

[5] Park SY, Park JD, kim JS, Baker J, Park JS. A novel Phellinus strain and the method for isolating $\beta-1,3$ glucomannan therefrom showing immune enhancing activity and antitumor activity. Korea Patent 1020070062608, filed June 26, 2007, Issued April 8, 2009.

[6] Kim JP, Back JA, Seo JJ, Yu JK. Anti-cancer agent comprising polysaccharide obtained from mycelia of Phellinus linteus and Adriamycin. Korea Patent 1020070002037, filed January 8, 2007, Issued July 17, 2008.

[7] Lacy EL, William AZ. Potentiation of Natural Killer Cells for Cancer Immunotherapy: A Review of Literature. Front Immunol. 2017;8:1061.

[8] Pere Santamaria. Cytokines and Chemokines in Autoimmune Disease: An Overview. Advances in Experimental Medicine and Biology, vol. 520. 2003;1-4.

[9] Victor CH, Raelene MM, Martha NB, Laura AM, Rachael K, Scott AB, Natalia M, Daniel RP, Gene HM, Jonathan AM. Distinct Contributions of Vaccine-Induced Immunoglobulin G1 (IgG1) and IgG2a Antibodies to Protective Immunity against Influenza. Clinical and Vaccine Immunology Sep 2006;13(9):981-990.

[10] Rhee YK, Han MJ, Park SY, Kim DH. In vitro and in vivo Antitumor Activity of the Fruit Body of Phellinus linteus. Korean Journal of Food Science and Technology. 2000:32(2);477-480.

[11] Lim JH, Kim SH, Park NH, Moon CJ, Kang SS, Kim SH, Shin DH, Kim JC. Acute and Chronic Antiinflammatory Effects of Phellinus linteus Water Extract in Rats. JBR. 2010;27-35.

[12] Kim HG, Yoon DH, Lee WH, Han SJ, Shrestha BS, Kim CH, Lim MH, Chang WC, Lim SY, Choi SG, Song WO, Sung JM, Hwang KC, Kim TW. Phellinus linteus inhibits inflammatory mediators by suppressing redox-based NF-KB and MAPKs activation in lipopolysaccharide-induced RAW 264.7 macrophage. Journal of Ethnopharmacology. 2007;307-315.

[13] Kim IH, Jin EJ, Lee JH. Antioxidant and Antimicrobial Activities of Cambodian Mushroom, Phellinus linteus. Environmental Mutagens and Carcinogens. 2006;26(2):41-44.

[14] Loris Z, Cristina P, Prisco M, Giuliana G, Stefano P, Laura G, Lucio C, Marco V. NK Cells and Cancer. The Journal of Immunology. 2007;178(7):4011-4016.

[15] Lodoen M, Lanier L. Viral modulation of NK cell immunity. Nat Rev Microbiol. 2005;3:59-69. 
[16] Vladimir J, Ivan S, Gordana K. A comparison of the NK cell cytotoxicity with effects of TNF-a against K-562 cells, determined by LDH release assay. Cancer Letters. 1999;138:67-72.

\section{Tables}

Table 1. Clinical trial process

Section

\begin{tabular}{|c|c|c|c|}
\hline \multirow[t]{2}{*}{ Screening } & Visit 1 & Visit 2 & Visit 3 \\
\hline & $\begin{array}{l}\text { (baseline } \\
\text { visit) }\end{array}$ & $\begin{array}{l}\text { (under } \\
\text { trial) }\end{array}$ & $\begin{array}{l}\text { (trial } \\
\text { end) }\end{array}$ \\
\hline $\begin{array}{l}(-2 w k \text { to } \\
0 \text { d) }\end{array}$ & $(0 \mathrm{~d})$ & $\begin{array}{l}\text { (4 wk } \pm 5 \\
\text { d) }\end{array}$ & $\begin{array}{l}\text { (8 wk } \pm 5 \\
\text { d) }\end{array}$ \\
\hline
\end{tabular}

Prepare a written consent form

$\sqrt{ }$

Inclusion/exclusion criteria evaluation

$\sqrt{ }$

Examining subject information

$\sqrt{ }$

Assign screening number

$\sqrt{ }$

Random assignment (the random number assigned)

Investigate past medical history and drug history

Physical examination

Vital signs

Clinical laboratory test (blood)

Pregnancy test

Observation of upper respiratory tract infection symptoms

Distribution of experimental/control foods

Effectiveness evaluation variable measurement

Check adverse events

Concomitant drug identification

Check compliance

Confirmation of cancellation and dropout criteria

$\sqrt{ }$

$\sqrt{ }$

$\begin{array}{lll}\sqrt{ } & \sqrt{ }\end{array}$

$\begin{array}{lll}\sqrt{ } & \sqrt{ }\end{array}$

V

$\checkmark$

$\sqrt{ }$

$\sqrt{ } \sqrt{ }$

$\sqrt{ } \sqrt{ }$

$\sqrt{ }$

$\begin{array}{llll}\sqrt{ } & \sqrt{ } & \sqrt{ }\end{array}$

$\begin{array}{llll}\sqrt{ } & \sqrt{ }\end{array}$

$\sqrt{ }$

-

$\sqrt{ } \sqrt{ }$

$\sqrt{ } \sqrt{ }$

$\sqrt{ } \sqrt{ }$

$\sqrt{ } \quad \sqrt{ }$


Figures

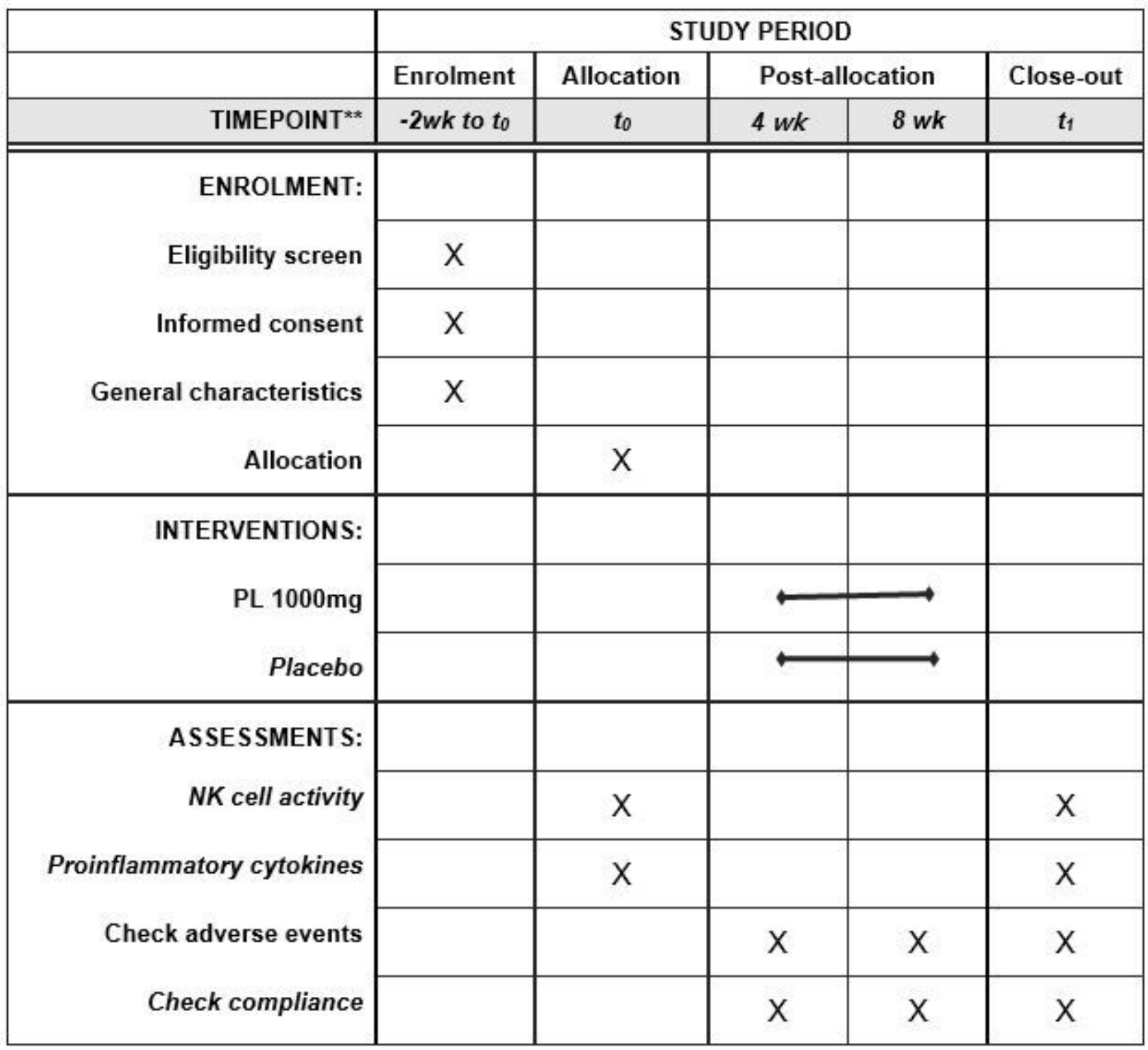

The " $X "$ refers to what is done in the given period.

Abbreviations: PL: Phellinus linteus; NK: natural killer

Figure 1

SPRIT figure showing schedule of enrolment, assessments, allocation for each patient.

\section{Supplementary Files}

This is a list of supplementary files associated with this preprint. Click to download.

- SPIRITchecklist.docx 